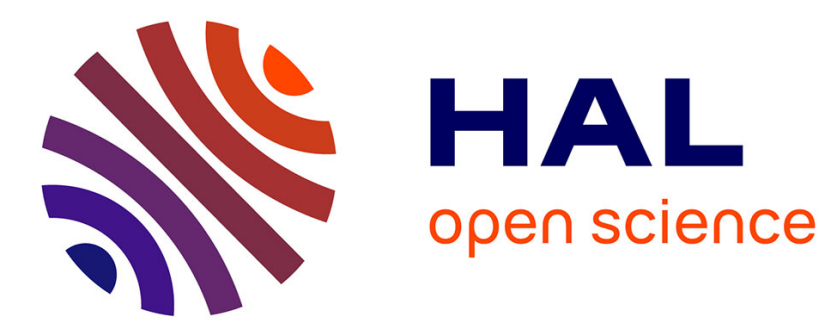

\title{
Imbibition on a porous layer: dynamical and mechanical characterization
}

\author{
Marguerite Leang, Ludovic Pauchard, Lay-Theng Lee, Frédérique \\ Giorgiutti-Dauphiné
}

\section{- To cite this version:}

Marguerite Leang, Ludovic Pauchard, Lay-Theng Lee, Frédérique Giorgiutti-Dauphiné. Imbibition on a porous layer: dynamical and mechanical characterization. Soft Matter, 2019, 15 (10), pp.2277-2283. $10.1039 / \mathrm{c} 8 \mathrm{sm} 02295 \mathrm{j}$. hal-02333955

\section{HAL Id: hal-02333955 https://hal.science/hal-02333955}

Submitted on 9 Nov 2020

HAL is a multi-disciplinary open access archive for the deposit and dissemination of scientific research documents, whether they are published or not. The documents may come from teaching and research institutions in France or abroad, or from public or private research centers.
L'archive ouverte pluridisciplinaire HAL, est destinée au dépôt et à la diffusion de documents scientifiques de niveau recherche, publiés ou non, émanant des établissements d'enseignement et de recherche français ou étrangers, des laboratoires publics ou privés. 


\section{Accepted Manuscript}

This article can be cited before page numbers have been issued, to do this please use: M. Leang, L.

Pauchard, L. Lee and F. Giorgiutti-Dauphiné, Soft Matter, 2019, DOI: 10.1039/C8SM02295J.

\section{Soft Matter}

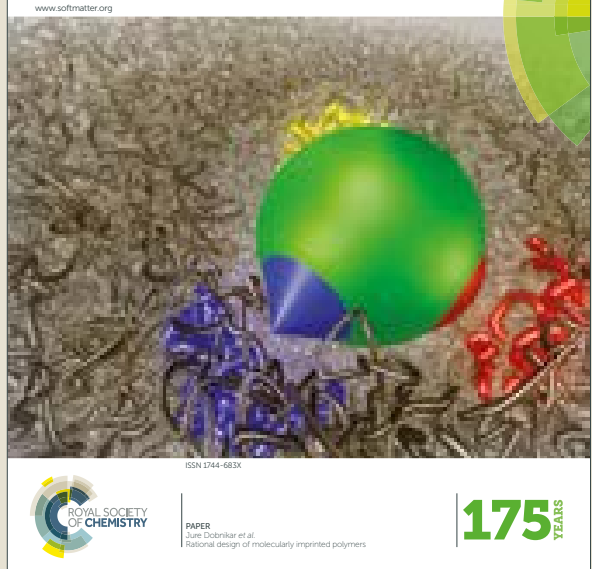

This is an Accepted Manuscript, which has been through the Royal Society of Chemistry peer review process and has been accepted for publication.

Accepted Manuscripts are published online shortly after acceptance, before technical editing, formatting and proof reading. Using this free service, authors can make their results available to the community, in citable form, before we publish the edited article. We will replace this Accepted Manuscript with the edited and formatted Advance Article as soon as it is available.

You can find more information about Accepted Manuscripts in the author guidelines.

Please note that technical editing may introduce minor changes to the text and/or graphics, which may alter content. The journal's standard Terms \& Conditions and the ethical guidelines, outlined in our author and reviewer resource centre, still apply. In no event shall the Royal Society of Chemistry be held responsible for any errors or omissions in this Accepted Manuscript or any consequences arising from the use of any information it contains. 


\title{
Journal Name
}

\section{ARTICLE TYPE}

Cite this: DOI: $10.1039 / x x x x x x x x x x$

\section{Imbibition on a porous layer: dynamical and mechani- cal characterization}

\author{
Marguerite Léang ${ }^{a, b}$, Ludovic Pauchard ${ }^{a}$, Lay-Theng Lee ${ }^{b}$ and Frédérique Giorgiutti- \\ Dauphiné ${ }^{\dagger \dagger}$
}

Received Date

Accepted Date

DOI: $10.1039 / x x x x x x x x x x$

Solvent penetration in porous layers was analyzed using dynamical and mechanical character zation. Spreading dynamics of a solvent drop in a porous substrate provided parameters of the porous medium such as permeability and porosity. These measurements are relevant for man; porous systems, for example paintings or porous varnishes and resins... We present direct visua izations of the drop as well as of the wet zone during the imbibition process and we evidence thre: distinct regimes. Experiments performed with various porous systems and different solvents hignlight a universal behavior. The mechanical properties during the imbibition process are deduce' 1 through indentation testing measurements. We show that solvent penetration is responsible fc. the appearance of a viscous component in the system. A characteristic time depending on th: solvent and on the porous medium is then deduced. The system recovers its initial mechanice. 1 properties and no swelling nor cracking is observed contrary to the case of paintings. This resu $t$ tends to prove that visco-plastic properties are required to observe swelling or cracking.

\section{Introduction}

The phenomenon of imbibition in porous media is widely encountered in industrial situations or in the domain of cultural heritage particularly when restauration operations are carried out to remove and to replace the varnish layer of a painting. Indeed, the most common treatment used by curators to remove varnish consists in using organic solvents ${ }^{1}$ applied to solubilize the old varnish and to remove it. Generally, when a solvent penetrates in a porous layer, it can cause swelling or inversely a decrease of volume after solvent evaporation, and consequently, it can induce additional stresses on the matter which lead to the formation of cracks. In addition, the solvent can modify the interactions between the components of the porous medium and consequently implies modifications of the mechanical properties of the porous layer.

To prevent these undesirable effects, one needs to study the dynamics of solvent penetration in a porous substrate and

\footnotetext{
${ }^{a}$ Laboratoire F.A.S.T, Univ. Paris-Sud, CNRS, Université Paris-Saclay, F-91405, Orsay, France; ${ }^{\dagger}$ Corresponding author, e-mail: fred@fast.u-psud.fr

${ }^{b}$ Laboratoire Léon Brillouin, UMR12 CEA-CNRS, Bât. 563 CEA Saclay, 91191 Gif sur Yvette Cedex, France.
}

the potential modifications induced on the matter such a dissolution, variations of the mechanical properties, modifications of interactions between various components of porou medium or changes in the stress field due to swelling for example

The aim of the presented work is to explain the physical mechanisms that come into play in imbibition processes and to identif: physical macroscopic parameters, as relevant parameters, to describe the imbibition process for various porous systems whit. could be paintings, varnish or resins.

To this end, we propose to study the imbibition process $f$ three different solvents in porous model systems formed after controlled drying of colloidal dispersions. The use of model systems has been motivated by the intent to deal with a well known and controlled system ${ }^{2}$. The problem of solvent imbibition process on a porous medium has been studied by various authors; the widely used geometry is the case of a liquid droplet deposited on a porous substrate. Different situations have been addressed: the case of a porous medium partly or entirely filled with liquid ${ }^{3,4}$, the case of a drop whose size is small or comparable to the size of the porous layer ${ }^{5}$. The wetting properties, the viscosity of the liquid or its volatility are the main parameters considered. Each pa- 
rameter will play a role on respectively the spreading of the drop, the viscous flow in the drop or/and the diffusion in the porous and the evaporation. Davis et al. ${ }^{6}$ and then Alleborn et al. ${ }^{7}$ used lubrication theory combined with Darcy's law to model spreading and sorption of a two-dimensional drop on a dry or saturated porous substrate. They obtained numeric profiles of the drop and of the wet zone, during the imbibition process. Some authors have included dynamic contact angle and contact angle hysteresis in their model or some employed Brinkman's equation to solve the flow in the porous medium ${ }^{8,9}$. Nevertheless, there are very few experimental visualizations, to our knowledge, of both the drop and the wet zone in the porous medium during the imbibition process ${ }^{10,11}$. In the present work, we propose an experimental study of the imbibition process based on direct visualization of simultaneously spreading and sorption of a drop on a porous substrate. The experimental profiles validate the numerical results obtained by solving both the lubrication and Darcy's equations in the work of Alleborn and Raszillier (2004). We performed experiments with three different solvents and two porous media and founded a universal behavior for the imbibition dynamics. In addition, we can estimate permeability of the porous substrate in two directions: parallel and perpendicular to the flow. We then present then indentation measurements conducted on the porous substrate before and after imbibition process to measure the impact of solvent penetration on the mechanical properties of the porous layer. Finally, we estimate dissolution of the porous media due to imbibition process.

\section{Imbibition dynamics}

\subsection{Material and methods}

The porous media of interest in this work are obtained during the drying of aqueous dispersions of silica colloidal particles: Ludox SM-30 and HS-40 commercially available from Sigma-Aldrich. The radius of silica particle is noted $a$ and the initial particle volume fraction is $\phi_{p, 0}$ (see Table 1). The $\mathrm{pH}$ value is in the range of $9-10$ to ensure that a high negative charge density is born by the particles; the DLVO theory is expected to apply and the dispersions are used without previous treatment. A liquid layer is formed by depositing a controlled volume of dispersion in a non-porous substrate (glass Petri dish), then the layer is left to dry from the free surface. The relative humidity is kept constant, $R H=54 \pm 2 \%$, at room temperature. Solvent evaporation concentrates the dispersion until the formation of a porous layer of approximately constant thickness in the centre of the container. The volume of dispersion initially deposited is chosen to obtain a solid layer thickness of around $1 \mathrm{~mm}$.

As drying proceeds, shrinkage is frustrated by adhesion with the substrate. It causes large tensile stresses which lead to the formation of cracks which divide the layer into polygonal adjacent fragments. These fragments have been characterized through

\begin{tabular}{|c|c|c|c|c|c|}
\hline System & \multicolumn{2}{|c|}{ Colloidal dispersion } & \multicolumn{2}{c|}{ Porous medium } \\
\hline & $\begin{array}{c}2 a \\
(\mathrm{~nm})\end{array}$ & $\phi_{p, 0}$ & $\mathrm{pH}$ & $1-\phi_{p}$ & $\begin{array}{c}\mathscr{K} \\
\left(\mathrm{m}^{2}\right)\end{array}$ \\
\hline SM-30 & 10 & $0.15 \pm 0.04$ & 9.9 & $0.33 \pm 0.02$ & $2.10^{-20}$ \\
HS-40 & 16 & $0.22 \pm 0.01$ & 9.8 & $0.31 \pm 0.03$ & $3.7 .10^{-20}$ \\
\hline
\end{tabular}

Table 1 Main properties of silica dispersions (particle diameter $2 a$ ) and porous media. $\phi_{p, 0}$ is the initial particle volume fraction of the dispersions. The volume fraction of the voids in the porous media is denoted $1-\phi_{p}$ with $\phi_{p}$, the particle volume fraction. The values have been determined by neutron imaging; $\mathscr{K}$ is the permeability calculated from Kozeny-Carman law ${ }^{12,13}$. Measurements uncertainties are averaged over three different samples.

\begin{tabular}{|c|c|c|c|c|}
\hline Solvent & $\begin{array}{c}M_{W} \\
\left(\text { g.mol }^{-1}\right)\end{array}$ & $\rho$ & $\begin{array}{c}\gamma \\
\left(m N . m^{-1}\right)\end{array}$ & $\begin{array}{c}\eta \\
(m P a . s)\end{array}$ \\
\hline water & 18 & 1 & 72.8 & 1 \\
ethyl acetate & 88.1 & 0.9 & 23.9 & 0.46 \\
glycerol & 92 & 1.26 & 63 & 1499 \\
\hline
\end{tabular}

Table 2 Main characteristics of the solvents used for imbibition processes at $T=20^{\circ} \mathrm{C} . M_{W}$ is the molecular weight; $\rho$, the density; $\gamma$, the surface tension and $\eta$, the dynamic viscosity ${ }^{14,15}$.

neutron imaging techniques to deduce the porosity $1-\phi_{p}$ of their structure, where $\phi_{p}$ is the particle volume fraction. The permeability $\mathscr{K}$ is deduced from the Kozeny-Carman law based on the size of the nanoparticles ${ }^{12,13}$. The main properties of the porous structure are reported in Table 1. One should note that we have considered fragments with no cracks as the presence of cracks will enhance and modify solvent penetration.

Three kinds of solvents are imbibed into the porous layers: water, ethyl acetate and glycerol. The choice of water and ethyl acetate has been motivated given their ordinary use by curators. Glycerol is not used in restoration but is a limiting case for the model due to its high viscosity and non-volatility. We have reported the main properties of the different solvents at $T=20^{\circ} \mathrm{C}$ in Table 2.

The experimental setup used to follow the dynamics of imbibition is presented in figure 1. A fragment of uniform thickness is selected. The thickness of a fragment is measured by successively focusing on the top (air-layer interface) and on the bottom (layer- substrate interface) of the deposited film in transmitted light. The accuracy of this method is to within $3 \mu \mathrm{m}$. Horizontality is adjusted by 3 screws on the sample holder. The experimental set-up allows us to image a fragment and provides both side and top view using two cameras (Marlin F131B, Allied Vision Technologies). Acquisition frequency is 1.875 frame per second (fps) for glycerol imbibition dynamics, whereas for water and ethyl acetate, we used a fast-speed camera Eo Sens 6546 CF 25-290 (Mikrotron $\mathrm{GmbH}$ ) with a 200 fps frequency. A drop of a given 
(a)

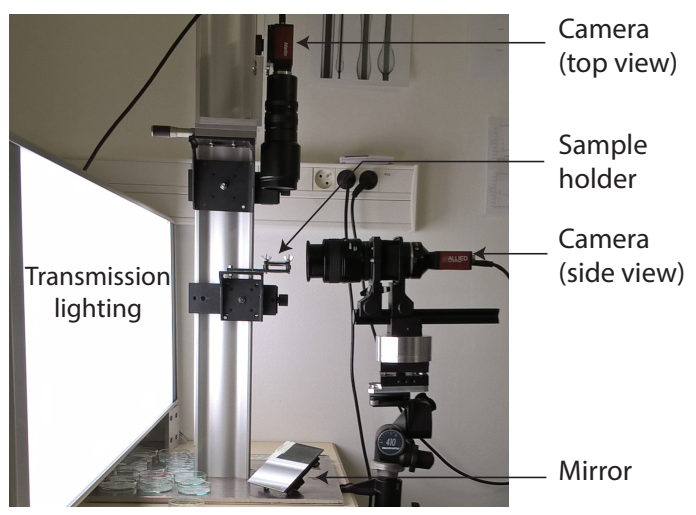

(b)

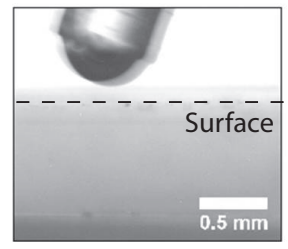

$\mathrm{t}=0 \mathrm{~s}$

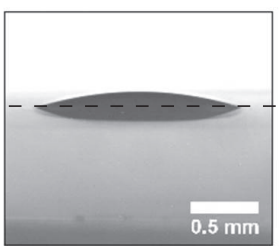

$t=0,2 s$

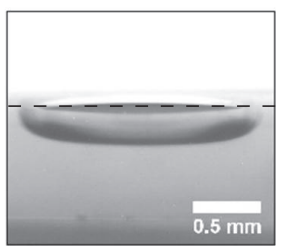

$t=5,2 s$

Fig. 1 (a) Experimental set-up composed of a sample holder, two cameras which acquire transmitted images. (b) Successive images during the imbibition of a glycerol drop on a porous system formed by drying Ludox HS-40.

volume is then deposited with a micropipette (Fisherbrand). The drop characteristic size is smaller than the capillary length ensuring an initial spherical cap shape for the sessile drops. The imbibition process takes place during a low duration in comparison with the evaporation timescale $t_{D}$, less than $<1 / 100$ (see Table 3 ). Thus, the whole drop that is deposited is assumed to penetrate into the porous layer. The drop and the wet area in the porous medium are illuminated by transmitted light, simultaneously. The two main advantages of the setup are the following. Firstly, the wet region can be followed with time by imaging successive profiles (see images in figure 1). Secondly, the axisymmetry of the deposited sessile drop and the wet region are easily followed using the top-view camera. Hence, the quantification of the profiles of both the sessile drop and the imbibed region reveals the conservation of the volume of liquid all along the imbibition process. This important point allows us to determine the volume of the imbibed region at each time.

The main parameters involved during the drop imbibition are reported in figure 2: the drop radius $r_{d}$ (the maximum radius is $r_{d \max }=r_{d}\left(t_{0}\right)$, reached at time $t_{0}$ ), the drop contact angle $\theta$ (the initial contact angle is noted $\theta_{0}$ ), the vertical penetration depth $z_{i m b}$ at the center of the drop. $h_{d}$ is the drop height at its center, and the initial drop height is $h_{d, 0}$.

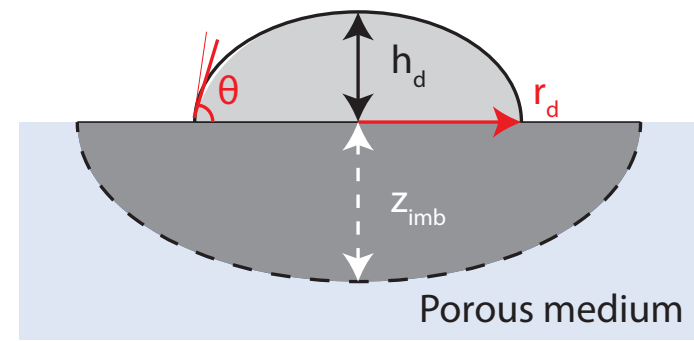

Fig. 2 Scheme of the drop on the porous substrate: definition of the experimentally measured lengths: $r_{d}$ is the drop radius, $h_{d}$ is its height at the drop centre, $\theta$ is the drop contact angle with the porous medium. Inside the porous medium, $z_{i m b}$ is the vertical penetration depth at the centre of the drop.

\subsection{Dynamics of drop imbibition}

The same scenario is observed for the three used solvents and both porous media: the imbibition process can be decomposed ir three distinct stages. In the first stage, the drop spreads on th: porous fragment; the drop radius increases whereas the conta angle decreases (images 1-3 in figure 3(a) for glycerol). In a second step, the surface area of the drop contact base is constar. the contact line is pinned by surface defects and the contac angle still decreases (see images from 3 to 4 in figure 3(a)! Simultaneously, the solvent starts penetrating in the porous Both radii, respectively of the drop and of the wet area are th. same. In the last stage, the drop is no more pinned and its radiu, decreases as well as its contact angle. The radius of the we area remains constant and equal to the maximum value of the drop radius whereas the vertical penetration increases (see figure 3(a), images from 4 to 10). At the end of the process, there is nc more solvent outside the porous and the wet area is axisymmetric

The time variations of the dimensionless drop radius, $r_{d} / r_{d \max }$. and dimensionless contact angle, $\theta / \theta_{0}$, are plotted in figure $4 \mathrm{f}$ different initial drop volumes. Note that time $t_{0}$ corresponds $\ldots$ the end of the spreading stage of the drop and is chosen as a tim reference.

We observe a brutal change of the slope for the drop radius correlated with the contact angle. These curves attest of a goc - ? reproducibility of the process. The data for experiments on two porous and with three solvents have been scaled with the Darcy's time, $t_{\text {Darcy }}$, defined as the time for the drop to penetrate in the porous medium, hence ${ }^{16}: t_{\text {Darcy }}=\eta h_{d}^{2}\left(t_{0}\right)\left(1-\phi_{p}\right) / \mathscr{K} P_{\text {cap }}$ with $\eta$ the viscosity of the solvent; $h_{d}\left(t_{0}\right)$, the height of the drop when the drop radius is maximum (at the end of the spreading stage $\left.t_{0}\right) ;\left(1-\phi_{p}\right)$ is the porosity of the medium; $\mathscr{K}$ is the permeability determined by neutron imaging (see values in Table 1) and $P_{c a p}$ is the capillary pressure which is the pressure of liquid in the pores of the system. Here, we take $P_{c a p} \sim \frac{\gamma}{a}$ where $\gamma$ is the solvent superficial tension and $a$, the particle ra- 
(a) Glycerol

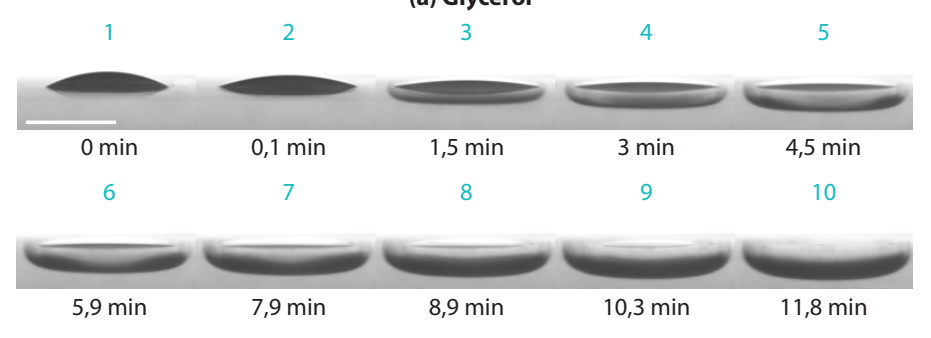

(b) Water

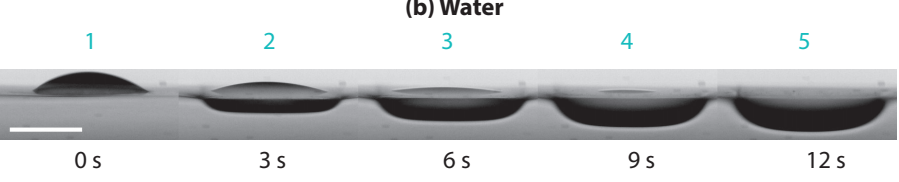

(c) Ethyl acetate

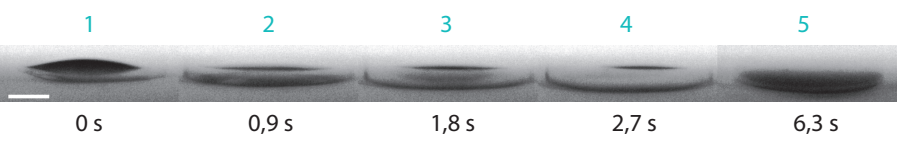

Fig. 3 Evolution of a drop of three solvent spreading and then imbibing porous fragment of Ludox HS-40 (particle radius $a=8 \mathrm{~nm}$ ). (a) The initial volume of glycerol drop: $0.1 \mu \mathrm{L}$. (b) The initial volume of water drop: 0.2 $\mu L$. (c) The initial volume of ethyl acetate: $0.08 \mu L$. Scale bar : $1 \mathrm{~mm}$.

dius $^{17}$. For water with HS-40 and SM-30 porous media, we found $9.3 * 10^{6} \mathrm{~Pa} \leq P_{\text {cap }} \leq 1.5 * 10^{7} \mathrm{~Pa}$ whereas for ethyl acetate with HS-40 and SM-30 porous media, $3 * 10^{6} \mathrm{~Pa} \leq P_{\text {cap }} \leq 5 * 10^{6} \mathrm{~Pa}$. Table 3 shows values of $t_{\text {Darcy }}$ for water and for ethyl acetate on HS-40 and SM-30 porous.

\begin{tabular}{|c|c|c|c|c|}
\hline Porous & HS-40 & SM-30 & \multicolumn{2}{|c|}{} \\
\hline Solvent & $\begin{array}{c}t_{\text {Darcy }} \\
(s)\end{array}$ & $\begin{array}{c}t_{\text {Darcy }} \\
(s)\end{array}$ & $\begin{array}{c}V_{E} \\
\left(\mathrm{~m}^{-1}\right)\end{array}$ & $\begin{array}{c}t_{D} \\
(s)\end{array}$ \\
\hline water & 10 & 11 & $5.10^{-8}$ & 2000 \\
ethyl acetate & 13 & 15 & $1.10^{-7}$ & 1000 \\
\hline
\end{tabular}

Table 3 Comparison of Darcy's time, $t_{\text {Darcy }}$ as a function of solvents and of porous media HS-40 and SM-30. $V_{E}$ is the evaporation rate. The drying timescales $t_{D}$ is defined as: $t_{D}=L_{i m b} / V_{E}$ where $L_{i m b}=100 \mu \mathrm{m}$ is a typical length for drop penetration in the porous media.

Note that for a kind of porous, Darcy's time of ethyl acetate is bigger than those of water. A possible explanation can be capillary condensation in the pores ${ }^{18}$. In fact, ethyl acetate evaporates faster than water and condensation of ethyl acetate vapour can take place in the void spaces. This can modify the wettability of the silica particle surface. Thus, ethyl acetate penetrates faster and easier in the porous media than water, leading to a bigger imbibition speed inside the porous media. The drying timescale $t_{D}$ is defined as the drop mean height divided by the solvent evaporation rate $V_{E}$, for constant relative humidity. This evaporation rate has been measured by the mass loss with time of a volume
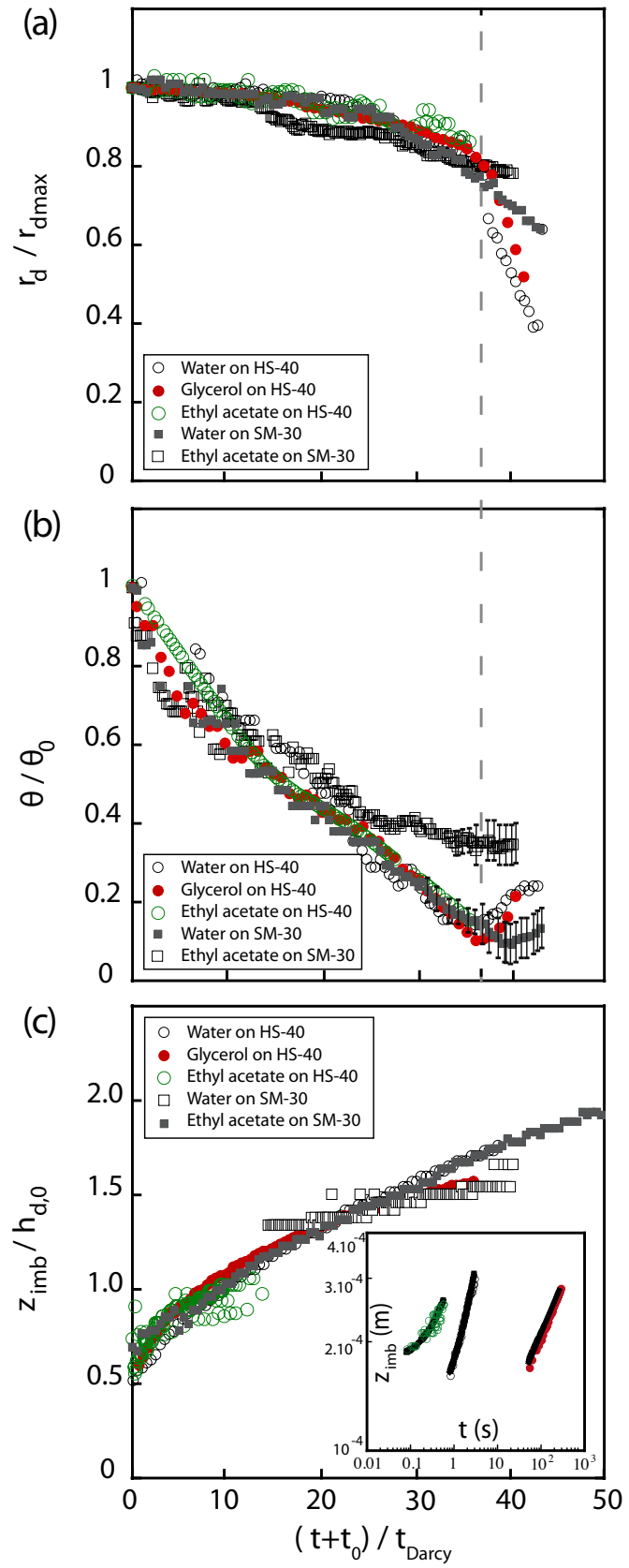

Fig. 4 (a) Time evolution of the dimensionless drop radius, $r_{d} / r_{d, \max }$, contact angle, $\theta / \theta_{0}$, and vertical penetration, $z_{i m b} / h_{d, 0}$, with the dimensionless time $\left(t+t_{0}\right) / t_{\text {Darcy }}$, where $t_{0}$ corresponds to the end of the drop spreading stage. Colours: different solvents penetrating in different porous media. Vertical dotted line: drop radius change is correlated to drop contact angle change. Inset : $z_{i m b}$ as a function of time, log-log scale. The initial contact angle, $\theta_{0}$, of Water, Glycerol and Ethyl acetate on the porous surfaces are $31 \pm 2^{\circ}, 20 \pm 2^{\circ}$ and $18 \pm 2^{\circ}$, respectively. 
of $5 \mathrm{~mL}$, in a Petri Dish, at ambient temperature and with a relative humidity of $50 \%{ }^{19}$. A typical length for drop penetration is $L_{i m b}=100 \mu m$. Table 3 shows the evaporation rate for water and ethyl acetate and the corresponding drying timescales.

All the curves collapse into a universal imbibition curve when plotted with the scaling time $t / t_{\text {Darcy }}$. A deviation is observed for dimensionless times about 10 ; at that time, the drop is very small and the resolution of the corresponding images do not provide enough precisions on the drop profiles. We have plotted as well, the height of the wet area $z_{i m b}$, scaled with the height of the initial drop $h_{d, 0}$. Again a universal behavior is evidenced.

The porosity values of the different porous media were deduced from neutron imaging techniques. We used a cold neutron imaging spectrometer. It allows performing radiography and tomography with fields of view ranging from 20 to $100 \mathrm{~mm}$ using a CMOS ANDOR NEO camera. Radiography can be performed with a fielc' of view of $200 * 400 \mathrm{~mm}^{2}$ by using a FUJI image plate. The inciden neutron beam has a diameter of $20 \mathrm{~mm}$, with a wavelength be tween 2 and 30 Angstrom. The spatial resolution is between 100 and 130 micrometers. The neutron beam passes over the sam. ple (a fragment of the porous medium, obtained by drying, witl a uniform thickness over the whole fragment) and we measure. the transmitted light on an image formed thanks to a scintilla tor and a camera. By dividing the transmitted light of a sample filled with water with the transmitted light of a dry sample, wi obtained the thickness of the sample traversed by the neutro1 beam and we deduce, knowing the total thickness of the sample, its porosity.

Another key parameter of the porous medium is the perme ability which can be deduced from two different ways: using th. Kozeny-Carman law or by direct measurements. This last methor is based on the dynamics of the imbibition front depicted by the spatiotemporal diagrams presented on figure 5 . We have records : with time the vertical and the horizontal dashed lines to produce. spatiotemporal diagrams of respectively the vertical and the ru dial imbibition front in the porous medium. We deduce the tem poral evolution of the wet area $z_{i m b}$ and its radial spreading $r_{i n}$, At the beginning of the imbibition process, evolutions of the fronts are linear and then become proportional to $\sqrt{t}$ to finally saturate. This behavior is similar to the dynamic of the capillary rise ${ }^{20}$ as the liquid penetration in the porous medium is due to a pressure gradient in the pores of the system, that is the capillary pressure. There are then three stages in the capillary rise dynamics which depend on the dominant physical mechanisms. The first stage is a regime dominated by the inertial forces, then the second regime is called the Washburn's regime where inertial forces become negligible compared to the viscous ones. The evolution of the imbibition front in the case of a porous medium is then given 
by $^{21,22}$ :

$$
z_{i m b}=\sqrt{\frac{\gamma \cos \left(\theta_{p}\right) r_{e f f}}{2 \eta T^{2}}} \sqrt{t}
$$

with $\gamma$, the solvent surface tension; $\theta_{p}$, the contact angle between solvent and particle surface $\left(\theta_{p}\right.$ is taken to be zero due to silica particle hydrophilicity); $r_{e f f}$, the effective radius of the porous and $T$, the tortuosity. The tortuosity is deduced from the porosity values through the relation $23,24,25: T=1 / \sqrt{1-\phi_{p}}$ and the permeability expresses as: $\mathscr{K}=\left(1-\phi_{p}\right) / 24 T r_{\text {eff }}^{2}$, which is given by Bear ${ }^{26}$. By fitting the curves (6f) representing the evolution of the imbibition front with time, with the Washburn's law, one can deduce the value of $r_{e f f}$. The results have been compared to values obtained with neutron imaging techniques (Table 4) and are equivalents (the experiment, in that case, consists of recording the imbibition front in a vertical fragment whose one end is in contact with a water bath) ${ }^{27}$. It turns out that the effective radius is always lower than the particle size, a, typically of factor 3 for the different colloidal systems we used. In the case of the sessile drop experiment, the fit of the curves in $6 \mathrm{f}$ with the Washburn's law, provides expressions for the two permeabilities in the two directions vertical and radial ones, respectively, $\mathscr{K}_{\text {vertical }}$ and $\mathscr{K}_{\text {radial }}$. The results are reported in Table 4 . The permeability in the radial direction $\mathscr{K}_{\text {radial }}$ is always higher than the one in the vertical direction $\mathscr{K}_{\text {vertical }}$. This observation can be related to results of previous work with the same systems in SANS experiments ${ }^{28}$. It has been shown that particles are closer in the direction of the drying flux, consequently, permeability of the final porous medium should be lower in this direction. These results are confirmed with our experimental values : $\mathscr{K}_{\text {vertical }} \leq \mathscr{K}_{\text {radial }}$. This suggests a non completely homogeneous porous medium, due to the vertical drying flux conducting to vertical compression of particles.

\section{Evolution of the mechanical properties before and after drop imbibition}

The mechanical properties of a porous medium strongly depend on its moisture content. This section is devoted to compare the elastic behavior of a porous medium before and after drop imbibition. In this way measurements using indentation testing (CSM Instrument Micro-Hardness Tester - Anton Paar) were investigated on HS-40 and SM-30 layers of $1 \mathrm{~mm}$ thick. A typical experiment consists in the measurement of an applied force, $F$, using a spherical indenter (Rockwell indenter of radius $R_{\text {indent }}=200 \mu \mathrm{m}$ ) as a function of the penetration depth, $p$, as shown in figure 6 . The maximum penetration depth of the indenter is always smaller than the solvent penetration depth $(\approx 500 \mu \mathrm{m})$, except at the end of solvent evaporation from the porous media. Assuming the material purely elastic within the limits of small deformations, the elastic response is well fitted using the Hertz contact law over a

\begin{tabular}{|c|c|c|c|c|}
\hline \multicolumn{5}{|c|}{ Neutron imaging } \\
\hline Porous media & $\begin{array}{c}a \\
(\mathrm{~nm})\end{array}$ & $\begin{array}{c}\text { Porosity } \\
1-\phi_{p}\end{array}$ & $\begin{array}{c}\kappa_{K C} \\
\left(\mathrm{~m}^{2}\right)\end{array}$ & $\begin{array}{c}\kappa_{\text {Bear }} \\
\left(\mathrm{m}^{2}\right)\end{array}$ \\
\hline Ludox SM-30 & 4.8 & 0.33 & $2.0 * 10^{-20}$ & $2.0 * 10^{-20}$ \\
Ludox HS-40 & 7.9 & 0.31 & $3.7 * 10^{-20}$ & $4.6 * 10^{-20}$ \\
\hline \multicolumn{6}{|c|}{ Sessile drop imbibition } \\
\hline & \multicolumn{6}{|c|}{ Vertical dynamics } & Radial dynamics \\
\hline Porous media & $r_{\text {eff }}$ vert & $\kappa_{\text {Bear }}^{\text {vert }}$ & $r_{\text {eff }}^{\text {radial }}$ & $\kappa_{\text {Bear }}^{\text {radial }}$ \\
& $(\mathrm{nm})$ & $\left(\mathrm{m}^{2}\right)$ & $\left(\mathrm{nm}^{2}\right)$ & $\left(\mathrm{m}^{2}\right)$ \\
\hline Ludox SM-30 & 1.66 & $2.7 * 10^{-20}$ & 3.0 & $8.0 * 10^{-20}$ \\
Ludox HS-40 & 1.80 & $2.9 * 10^{-20}$ & 4.0 & $1.5 * 10^{-19}$ \\
\hline
\end{tabular}

Table 4 Comparison or mean pore radius determined by Washburn's law for vertical and radial imbibition dynamics of a drop inside 2 porous media and mean pore radius determined by neutron imaging. $a$ : particle radius composing the porous media, $\kappa_{K C}$ : Kozeny-Carman permability, $\kappa_{\text {Bear }}$ : Bear's law permeability. $r_{\text {eff }}{ }^{\text {vert }}, \kappa_{\text {Bear }}{ }^{\text {vert }}$ : vertical imbibition, mean pore radius and Bear's permeability respectively; $r_{\text {eff }}{ }^{\text {radial }}, \kappa_{\text {Bear }}{ }^{\text {radial }}$ : radial imbibition, mean pore radius and Bear's permeability respectively.

range of indentation depth such as $p<1.2 \mu m$ (figure 6). Hence, according to the classical Hertzian model, the applied indentation force $F$ is determined by ${ }^{29}$ :

$$
F=\frac{4 \sqrt{R_{\text {indent }}}}{3} \frac{E}{1-v^{2}} p^{\frac{3}{2}}
$$

with the Poisson ratio $v=0.2^{30}$. Typical elastic behavior of a porous medium before drop imbibition is shown in figure 6 . It has to be noted, the water moisture content in the porous layer is not zero since the humidity rate of the surroundings is finite. Within the first minutes following the drop deposition, measurements using indentation testing do not allow us to estimate elastic response. Once the drop is totally penetrated inside the layer, measurements are possible in the wet region. The load-displacement measurements are then characteristic of a low elastic modulus. Further measurements show recovery with time of the elastic behavior of the porous layer (figure 6). Figure 6 reports the case of ethyl acetate imbibition into HS-40 porous layers. Similar behaviors are obtained with SM-30 porous layers and with water imbibition, the timescales involved being different. The elastic moduli which are deduced from load-displacement curves and equation 2, are shown in figure 7. Using the poroelastic theory it has been shown that the drying stress linearly increases with time $^{31}$. However, this model stated a constant elastic modulus with time. The recovery with time of the elastic behavior can be pointed out by the following simple arguments. Consider $X$ being the current moisture content of the porous medium after drop imbibition. $E_{0}$ is the elastic modulus of the medium without solvent content addition, the linearity between stress, $\sigma$, applied to the porous medium and its strain, $\varepsilon_{0}$, writes: $\sigma=E_{0} \varepsilon_{0}$ in the condi- 
$0 \mathrm{~ms}$

4

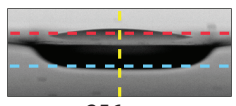

$256 \mathrm{~ms}$

7

600 (a)

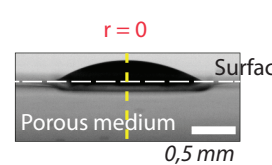

$r$

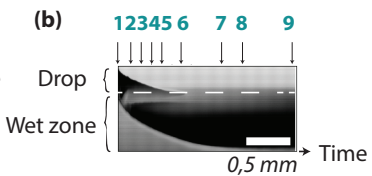

(c)

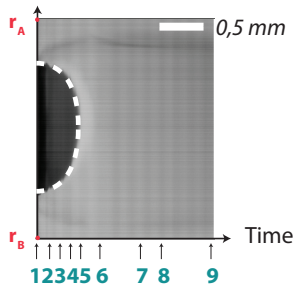

(d)

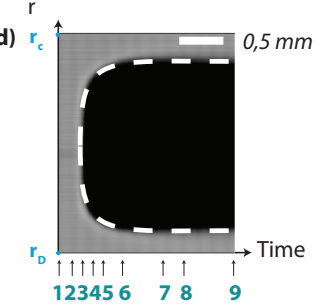

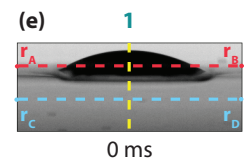
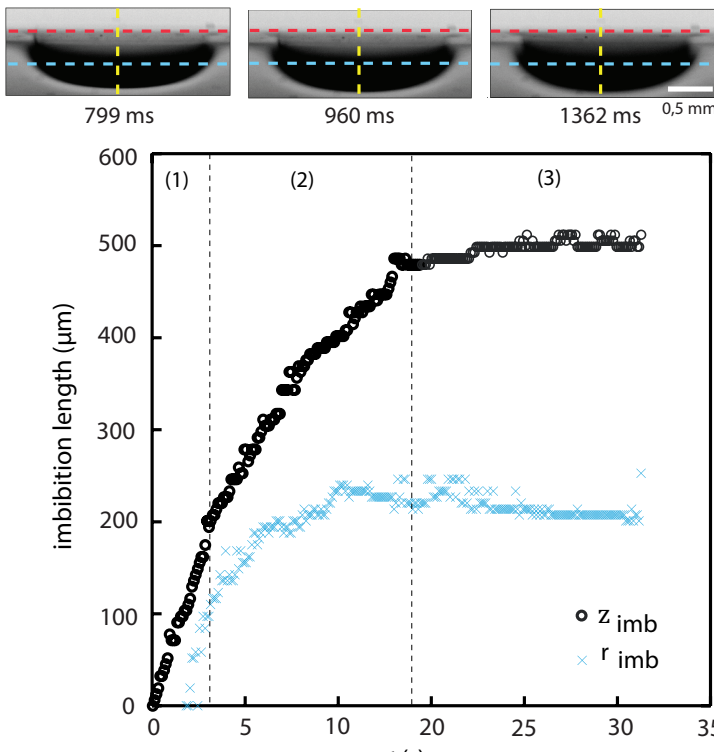

$\mathrm{t}(\mathrm{s})$

Fig. 5 Time evolution of vertical and the radial fronts. (a) Side view of a drop deposited on the surface of a porous medium. (b) Spatio-temporal diagram along the yellow dashed line, $r=0$, in (a). (c),(d)

Spatio-temporal diagrams obtained along the red and the blue horizontal lines represented in (e), i.e in the drop, and in the wet zone.

(e) Images of the drop imbibition at different times. The numbers in (b)-(d) correspond to the images in (e). Scale bar: $0.5 \mathrm{~mm}$. (f) Position for the imbibition front with time in vertical and radial directions; the Washburn regime corresponds to the zone (2).
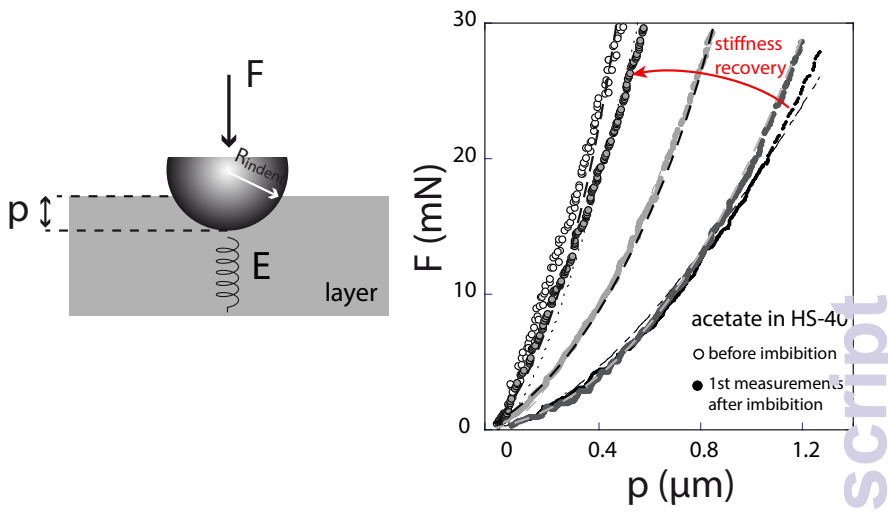

Fig. 6 Spherical indentation load-displacement (applied force $F$ vs. penetration depth $p$ ) in HS-40 porous layers. The present test shows a series of measurements performed before imbibition (o). Then each series of dots correspond to an indentation load-displacement performed after ethyl acetate imbibition and during the stiffness recovery. The measurements (dots) are fitted by dashed lines accordina to equation 2.

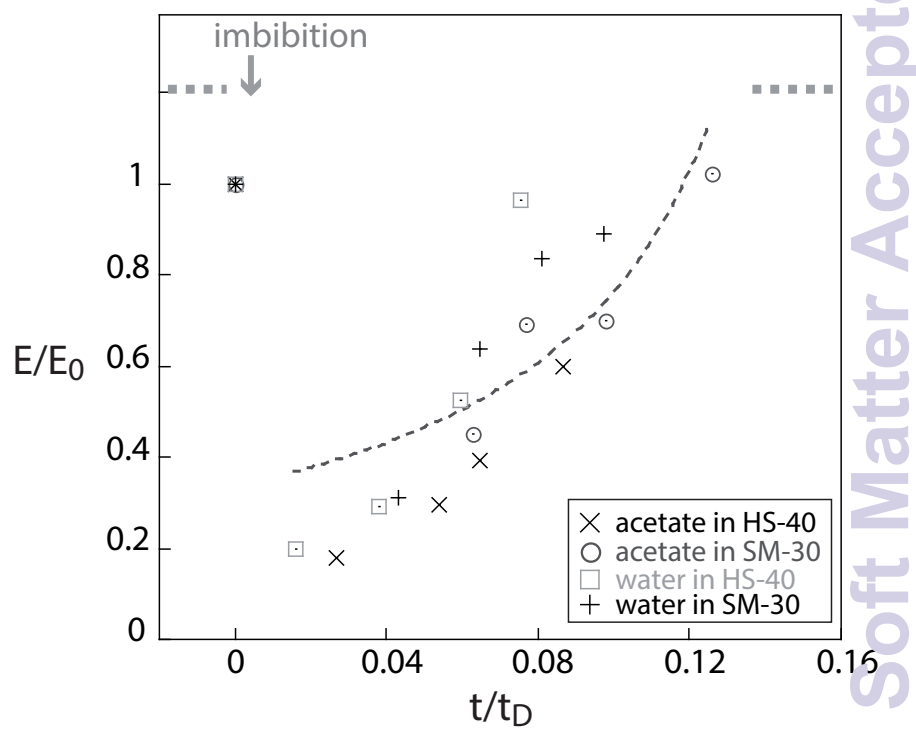

Fig. 7 Evolution of the dimensionless elastic modulus, $E / E_{0}$, as a function of the dimensionless time, $t / t_{D}$, for different porous media (HS-40 and SM-30) after imbibition by ethyl acetate or water. $E_{0}$ is the elastic modulus before drop imbibition $\left(E_{0}=1.1 \pm 0.6 \mathrm{GPa}\right)$; $t_{D}$ is the drying timescale. The moment of the imbibition process is indicated by the arrow. The dashed line corresponds to the theoretical predictions according to equation 3 .

tions of applicability of the Hooke's law. We observe no swelling of the material when water penetrated into the porous media, 
however, there is an additional deformation $\varepsilon(X)$ due to imbibition that we assumed to be proportional to the quantity of solvent which has penetrated in the porous medium. The degree of solvent saturation affects thus the elastic response of the porous medium and this results in larger strain, $\varepsilon+\varepsilon(X)$, and specific elastic modulus, $E(X)$, such as: $\sigma=E(X)\left(\varepsilon_{0}+\varepsilon(X)\right)$. The solvent molecules and silica structures are assumed to be incompressible. Consequently, the strain resulting from the moisture content can be written: $\varepsilon(X)=\varepsilon_{0} \mathscr{V} X$ where $\mathscr{V}$ is the volume per solvent molecule. The evaporation rate of the solvent is assumed to be constant during the main solvent removal period and is defined as: $V_{E}=-\frac{\mathscr{V}}{S_{0}} \frac{d X}{d t}$ where $S_{0}$ is the evaporation surface. Finally the experimental results can be fitted by the law:

$$
E(X) / E_{0}=1 /\left(1+\alpha\left(1-t / t_{D}\right)\right)
$$

where $\alpha$ is a coefficient of the influence of the moisture content on the elastic properties of the material ${ }^{32}$. This highlights the effect of solvent evaporation in the timing recovery of the elastic behavior of a porous medium previously imbibed. In order to observe swelling and cracking as a result of solvent imbibition and solvent evaporation, visco-plastic properties seem to be required ${ }^{32,33,34}$.

\section{Conclusion}

We investigated the imbibition dynamics of three different solvents on two porous media. These porous media were characterized previously by neutron imaging. We implemented a set-up which permitted us to (i) verify the axisymmetry of deposited drops from a top view; (ii) to follow the drop profiles and the liquid front profiles inside the porous media. We described the imbibition dynamics by quantifying the drop radius, the drop contact angle, and the vertical penetration depth at the centre of the drop. A relevant characteristic time for imbibition is determined to be Darcy's timescale: a universal behavior for different solvents and porous media is shown. These dynamics are completed with mechanical properties of porous media, before and after imbibition. Particularly, viscoelasticity was measured by creep measurements and show different characteristic times for water and ethyl acetate. Moreover, the fitting of micro-indentation loading curves with the Hertz's contact law permitted the determination of Young's modulus of the porous media before and after drop imbibition. A recovery of mechanical properties was evidenced as solvent evaporated. This result tends to prove that visco-plastic properties are required to observe swelling or cracking.

\section{Acknowledgment}

We acknowledge financial support through the Funding agency: Investissement d'Avenir LabEx PALM (Grant number : ANR-10LABX-0039). We also acknowledge Laboratory Léon Brillouin
(LLB) and CEA Saclay for giving neutron beamtime on IMAGINE (G3 bis), neutron imaging spectrometer. Many thanks go to E. Rabot (LLB) and F. Ott (LLB) for their help and advice on IMAGINE. We also thank S. Kwok and B. Cabane for fruitful discussions. We acknowledge F. Gibert (LLB), J. Amarni, A. Aubertin, L. Auffray and R. Pidoux (FAST-University Paris Sud) for engineering and technical support and Sofia Almagro Carrasco.

\section{References}

1 N. Stolow, Recent advances in conservation: contributions to the IIC Rome Conference, 1961, pp. 84-88.

2 F. Giorgiutti-Dauphiné and L. Pauchard, Journal of Applied Physics, 2016, 120, 065107.

3 V. M. Starov, S. R. Kosvintsev, V. D. Sobolev, M. G. Verlarde and S. A. Zhdanov, J. Colloid Interface Sci., 2002, 246, 372.

4 V. Starov, S. Zhdanov, S. Kosvintsev, V. Sobolev and M. Velarde, Advances in Colloid and Interface Science, 2003, 104, 123-158.

5 V. M. Starov, S. A. Zhdanov and M. G. Velarde, Langmuir, 2002, 18, 9744-9750.

6 S. H. Davis and L. M. Hocking, Phys. Fluids, 2000, 12, 1646.

7 N. Alleborn and H. Raszillier, Chemical Engineering Science, 2004, 59, 2071-2088.

8 M. Hilpert and A. Ben-David, International Journal of Multiphase Flow, 2009, 35, 205-218.

9 Wetting and spreading dynamics, ed. C. Press, Starov, V. M. and Velarde, M. G. and Radke, C. J., 2007.

10 S. Ogawa and J. Nakamura, J. Opt. Soc. Am. A, 2015, 32, 2397-2406.

11 J. Lee, A. Radu, P. Vontobel, D. Derome and J. Carmeliet, Journal of Colloid and Interface Science, 2016, 471, 59-70.

12 J. Kozeny, Über kapillare leitung des wassers im boden: aufstieg, versickerung und anwendung auf die bewässerung, HölderPichler-Tempsky, 1927.

13 P. C. Carman, Chemical Engineering Research and Design, 1997, 75, S32-S48.

14 CRC Handbook of Chemistry and Physics, 56th Edition, ed. E. R. Weast, CRC Press, 1975-1976.

15 M. L. Sheely, Industrial \& Engineering Chemistry, 1932, 24, 1060-1064.

16 Z. Liu, Y. Wang, F. J. Muzzio, G. Callegari and G. Drazer, Langmuir, 2017, 33, 56-65.

17 W. P. Lee and A. F. Routh, Langmuir, 2004, 20, 9885-9888.

18 L. Bacri and F. Brochard-Wyart, The European Physical Journal E, 2000, 3, 87-97.

19 F. Boulogne, F. Giorgiutti-Dauphiné and L. Pauchard, Oil Gas 
Sci. Technol. - Rev. IFP Energies nouvelles, 2014, 69, 397 - 404. 20 J. Bico, PhD thesis, Université Paris VI, 2000.

21 F. A. L. Dullien, Porous Media: Fluid Transport and Pore Structure, San Diego, CA :Academic press, 1979.

22 R. Hassanein, H. O. Meyer, A. Carminati, M. Estermann, E. Lehmann and P. Vontobel, Journal of Physics D: Applied Physics, 2006, 39, 4284.

23 Y. Bernabé and A. Revil, Geophysical Research Letters, 1995, 22, 1529-1532.

24 P. W. Glover, I. I. Zadjali and K. A. Frew, GEOPHYSICS, 2006, 71, F49-F60.

25 P. W. Glover and E. Walker, GEOPHYSICS, 2009, 74, E17-E29.

26 J. Bear, Dynamics of fluids in porous media, Dover Publication Inc., New York, 1988.

27 M. Léang, PhD thesis, paris-Saclay University, 2017.
28 F. Boulogne, L. Pauchard, F. Giorgiutti-Dauphiné, R. Botet, R. Schweins, M. Sztucki, J. Li, B. Cabane and L. Goehring, EPL (Europhysics Letters), 2014, 105, 38005.

29 A. C. Fischer-Cripps, Nanoindentation, Springer New York, New York, NY, 2004, pp. 1-20.

30 E. Di Giuseppe, A. Davaille, E. Mittelstaedt and M. François, Rheol. Acta, 2012, 51, 451-465.

31 Giorgiutti-Dauphiné, F. and Pauchard, L., Eur. Phys. J. E, 2014, $37,39$.

32 S. Kowalski, Chemical Engineering Journal, 2002, 86, 145151.

33 S. M. Wiederhorn, T. Fett, J.-P. Guin and M. Ciccotti, Interna tional Journal of Applied Glass Science, 2013, 4, 76-86.

34 M. Liu and C. Chen, International Journal of Solids and Struc. tures, 2015, 63, $32-38$. 